\section{PO 8569 INNOVATIVE DOMESTIC FINANCING FOR HEALTH RESEARCH \& DEVELOPMENT IN THE EAST AFRICAN COMMUNITY}

${ }^{1}$ Jean De Dieu Ngirabega, ${ }^{1}$ Novat Twungubumwe, ${ }^{2}$ Nandan K Pulakkal, ${ }^{3}$ Prince N Bahati, ${ }^{3}$ Anatoli Kamali, ${ }^{1}$ Gibson S Kibiki. ${ }^{1}$ East African Health Research Commission, Arusha, Tanzania; ${ }^{2}$ Future Options Consulting Ltd, Kampala, Uganda; ${ }^{3}$ International AIDS Vaccine Initiative, New York, USA

\subsection{6/bmjgh-2019-EDC.144}

Background Access to domestic financial resources is a prerequisite for strengthening health research and development (R\&D). Therefore, the East African Health Research Commission (EAHRC) commissioned a study to assess the financial needs of the East African Community (EAC) region and propose innovative domestic financing mechanisms for $\mathrm{R}$ and $\mathrm{D}$ in East Africa.

Methods This study used a four-pronged approach as follows: a desk review of secondary data, followed by a survey to collect quantitative data from health R\&D organisations and relevant ministries, followed by key informant interviews and, finally, a validation workshop. The study used 2014-2015 as the baseline year.

Results Only 51 out of 160 organisations responded to the survey. Using triangulation of desk reviews, national budgets documents and reported organisational budgets, the annual investment in health R\&D in the EAC is estimated at USD 301.71 million of which $86 \%$ is financed from external sources. The share of health R\&D financing in the GDP and health budget stood at $0.21 \%$ and $1.27 \%$ respectively, while the share of domestic financing of health R\&D to GDP was as low as $0.03 \%$

The innovative domestic financing options suggested included: allocation of $10 \%$ of the USD 560 million of the sin-taxes collected; taxing $1 \%$ of the estimated USD 3 billion from inward remittances; fundraising for at least $2.5 \%$ of the USD 18.67 private sector investment in corporate social responsibility; issuing social impact bonds and the EAC Health Research Fund with an estimated annual performance of USD 20 million.

Conclusion In order to sustain health $R \& D$ investments in EAC, the EAHRC proposes to develop a 10 year domestic financing roadmap using a strategic mix of tax- and non-taxbased innovations.

\section{PO 8571 CYTOKINE PROFILE IN ASYMPTOMATIC SCHOOL CHILDREN CO-INFECTED WITH HELMINTHS AND PLASMODIUM FALCIPARUM IN IBADAN, SOUTH-WEST NIGERIA}

${ }^{1}$ George Ademowo*, ${ }^{2}$ Olawunmi Rabiu, ${ }^{2}$ Ganiyu Arinola, ${ }^{2}$ Catherine 0 Falade. ${ }^{1}$ University of Ibadan, Nigeria; ${ }^{2}$ College of Medicine, University of Ibadan, Nigeria

\subsection{6/bmjgh-2019-EDC.145}

Background Intestinal helminths and malaria are among the most prevalent infectious diseases in the tropics. The effect of co-infections on immune response is not clearly understood. We therefore investigated the immune response profile in children with and without symptoms.

Methods A total of 78 afebrile school children (20 helminth/ malaria-co-infected, 17 helminth-infected, 19 malaria-infected and 22 uninfected) and 75 febrile children (14 helminth/ malaria-co-infected, 16 helminth-infected, 20 malaria-infected and 25 uninfected) were recruited into the study. Helminths were screened using Kato Katz method while malaria parasite screening was done using Giemsa-stained thick blood films. Circulating TNF- $\alpha$, IFN- $\gamma$, IL-1, IL-10 and IL- 6 concentrations were assessed by ELISA from serum samples. Data were analysed using analysis of variance.

Results Among the afebrile school children, IL-10 was significantly increased in helminth-infected children compared with helminth/malaria-co-infected, malaria-infected and uninfected groups $(p<0.05)$. IFN- $\gamma$ was significantly elevated in malaria and malaria/helminth-co-infection relative to helminth alone $(p<0.05)$. IL-1 level was significantly higher in single infection of helminth and malaria relative to co-infection and the uninfected groups $(p<0.05)$. An insignificant difference was observed for IL- 6 and TNF- $\alpha$ concentrations across all the four groups while among febrile children. IL-6 was significantly increased among helminth alone and helminth/malariaco-infection relative to malaria-infected group $(p<0.05)$. IL-10 was significantly elevated in co-infected group compared with helminth- or malaria-infected group while TNF- $\alpha$ was significantly increased in helminth and helminth-malaria co-infection compared with uninfected or malaria-infected group $(\mathrm{p}<0.05)$. IFN- $\gamma$ level was insignificant in the infection groups relative to uninfected group $(\mathrm{p}<0.05)$; IL-1 level similar across the groups.

Conclusion Helminth infection seems to upregulate the Th2 immune response among children with symptomatic uncomplicated malaria while there were no significant changes in Th immune response among afebrile children.

\section{PO 8572 CAUSES OF HOSPITALISATION AND MORTALITY IN CHILDREN UNDER 5 YEARS OLD, NATIONAL HOSPITAL OF GUINEA-BISSAU, 2015-2017}

Isaquel B Silva, Christian B Øland. Bandim Health Project, Bissau, Guinea - Bissau

\subsection{6/bmjgh-2019-EDC.146}

Background Studying the causes of hospitalisation is useful to understand the profile of illness and identify the most effective interventions. Guinea-Bissau was projected to reduce the under-5 mortality rate from 200 to $80 / 1000$ live births (2005-2015); and the causes of the deaths were attributed: neonatal, pneumonia, malaria and diarrhea. In 2014 the mortality rate was 55/1000 live births in Guinea-Bissau, and malaria, diarrhea and respiratory infection were the main causes of illness. The present study aims to describe the main causes of hospitalisation and death in children under 5 years in the paediatric clinic of the 'Simão Mendes' National Hospital.

Method Descriptive and retrospective study, with search of data from health care records. STATA and Microsoft Excel programmes were used for data analysis and cleaning. Cases defined as: children under 5 years of age, diagnosed from 2015 to 2017.

Results In 17,250 cases of hospitalisation, the overall lethality rate for 2015-2017 was calculated at 7.5\%. There was an increase in the lethality rate $(10.8 \%)$ in 2017. Among the main causes of hospitalisation were gastrointestinal infection (26.9\%), malaria (23\%), respiratory infection (17.6\%) and septicaemia $(16.1 \%)$. Septicaemia is the disease with the highest lethality rate during these three years (18\%), the case fatality rate due to gastrointestinal infection in 2017 (7.7\%) was 